JOURNAL OF THE AMERICAN MATHEMATICAL SOCIETY

Volume 19, Number 3, Pages 717-736

S 0894-0347(05)00517-5

Article electronically published on December 21, 2005

\title{
A SOLUTION TO THE L SPACE PROBLEM
}

\author{
JUSTIN TATCH MOORE
}

This paper is dedicated to Stevo Todorcevic for teaching me how to traverse $\omega_{1}$ and for his inspirational [23].

\section{INTRODUCTION}

In 23, Todorcevic gives a survey of basis problems in combinatorial set theory, listing nine theorems and six working conjectures 1 - all in the presence of PFA including the following three of interest to us here:

Conjecture 1 (Todorcevic; 23]). If $R$ is a binary relation, then either $R \leq \aleph_{0} \cdot \omega_{1}$ or $\left[\omega_{1}\right]^{<\aleph_{0}} \leq R$.

Conjecture 2 (Hajnal, Juhasz; [5]). If $X$ is a regular Hausdorff space, then the following are equivalent:

(i) $X$ is hereditarily separable;

(ii) $X$ is hereditarily Lindelöf;

(iii) $X$ does not contain an uncountable discrete subspace.

Conjecture 3 (Gruenhage; 3). The uncountable regular Hausdorff spaces have a three element basis consisting of a set of reals of cardinality $\aleph_{1}$ with the metric, the Sorgenfrey, and the discrete topology.

According to the survey article [7], Kunen formulated the partition relation

$$
\omega_{1} \rightarrow\left(\omega_{1},\left(\omega_{1} ; \omega_{1}\right)\right)^{2},
$$

while studying Conjecture 2 in the 1970s. He showed that it would imply a positive resolution of Conjecture 2 and asked whether it is consistent. Afterward Laver showed that under $\mathrm{MA}_{\aleph_{1}}$ this partition relation is equivalent to the formally weaker partition relation

$$
\omega_{1} \rightarrow\left(\omega_{1} ; \omega_{1}\right)_{2}^{2} .
$$

Around the same time, Galvin showed that the positive square bracket relation $\omega_{1} \rightarrow\left[\omega_{1}\right]_{5}^{2}$ implies $\omega_{1} \rightarrow\left(\omega_{1} ; \omega_{1}\right)_{2}^{2}$.

Received by the editors January 8, 2005.

2000 Mathematics Subject Classification. Primary 54D20, 54D65, 03E02, 03E75; Secondary $54 \mathrm{~F} 15$.

Key words and phrases. L space, negative partition relation, Tukey order, hereditarily Lindelöf, non-separable, basis.

The research presented in this paper was funded by NSF grant DMS-0401893.

1 Many of these were not phrased as conjectures by their authors but rather as problems. Moreover, they were often phrased as "Is it consistent that ...?" rather than "Does PFA imply that ...?". 
In [19] Todorcevic showed that $\omega_{1} \nrightarrow\left[\omega_{1}\right]_{5}^{2}$ by employing a new technique - the method of minimal walks - which has also proved useful in many other applications (see 24]). This technique will be employed in this paper where I will prove the following results which refute $\omega_{1} \rightarrow\left(\omega_{1} ; \omega_{1}\right)_{2}^{2}$ and Conjectures 13 .

Theorem 1.1. There is a function $f:\left[\omega_{1}\right]^{2} \rightarrow \omega_{1}$ such that whenever $A, B \subseteq \omega_{1}$ are uncountable and $\xi<\omega_{1}$ there are $\alpha$ in $A$ and $\beta$ in $B$ such that $\alpha<\beta$ and $f(\alpha, \beta)=\xi$. In particular $\omega_{1} \nrightarrow\left(\omega_{1} ; \omega_{1}\right)_{2}^{2}$.

Theorem 1.2. There is a family $\mathscr{F}$ of binary relations which contains an antichain of cardinality $2^{\aleph_{1}}$, is downwards directed, and such that for all $R$ in $\mathscr{F}, R \not \aleph_{0} \cdot \omega_{1}$ and $\left[\omega_{1}\right]<\aleph_{0} \not \leq R$.

Theorem 1.3. There is a non-separable, hereditarily Lindelöf subspace $\mathscr{L}$ of $\mathbb{T}^{\omega_{1}}$. Moreover the closure in $\mathbb{T}^{\omega_{1}}$ of any uncountable subset of $\mathscr{L}$ contains a canonical homeomorphic copy of $\mathbb{T}^{\omega_{1}}$.

Theorem 1.4. Any basis for the uncountable topological spaces has cardinality strictly greater than $\aleph_{1}$.

It is worth noting that it was known early on that the existence of an L space alone gives a refutation of these conjectures. The above results, however, take the failures of these conjectures a step further and require slightly different combinatorial refinements.

The theorems in this paper are consequences of an analysis of coherent sequences of finite-to-one functions $\left\langle e_{\beta}: \beta<\omega_{1}\right\rangle$ and lower trace functions $L:\left[\omega_{1}\right]^{2} \rightarrow$ $\left[\omega_{1}\right]^{<\omega_{1}}$. These are combinatorial objects which can be routinely constructed using the method of minimal walks. If we let $\operatorname{osc}(\alpha, \beta)$ denote the number of changes in the relation between corresponding entries of $e_{\alpha}\left\lceil L(\alpha, \beta)\right.$ and $e_{\alpha}\lceil L(\alpha, \beta)$ from $\leq$ to $>$, then the main result of the paper can be stated as follows.

Theorem 1.5. For every $\mathscr{A} \subseteq\left[\omega_{1}\right]^{k}$ and $\mathscr{B} \subseteq\left[\omega_{1}\right]^{l}$ which are uncountable families of pairwise disjoint sets and every natural number $n$, there are a in $\mathscr{A}$ and $b_{m}$ $(m<n)$ in $\mathscr{B}$ such that for all $i<k, j<l$, and $m<n$ :

$$
\begin{aligned}
a & <b_{m}, \\
\operatorname{osc}\left(a(i), b_{m}(j)\right) & =\operatorname{osc}\left(a(i), b_{0}(j)\right)+m .
\end{aligned}
$$

This can be likened to the following two results of Todorcevic. The first is used to draw a number of conclusions about Conjecture 2] in 21. The second yields one of many proofs in Section 4 of [19] that $\omega_{1} \nrightarrow\left[\omega_{1}\right]_{\omega}^{2}$. See [19] and Section 2 below for undefined notation 2

Theorem 1.6. 20. If $X$ and $Y$ are unbounded and countably directed in $\left(\omega^{\omega},<^{*}\right)$ and consist of monotonic functions, then there is a natural number $l$ such that for all $n$ there are $x$ and $y$ in $X$ and $Y$ respectively such that

$$
\operatorname{osc}(x, y)=n+l \text {. }
$$

Theorem 1.7. 19] If $X$ is an uncountable subset of $\omega_{1}$, then the set of all values

$$
\operatorname{osc}(\operatorname{Tr}(\zeta, \alpha), \operatorname{Tr}(\zeta, \beta))
$$

for $\alpha \neq \beta$ in $X$ with $\zeta=\Delta(\alpha, \beta)$ contains arbitrarily long intervals of integers.

${ }^{2}$ What I am referring to as $\operatorname{Tr}(\alpha, \beta)$ is denoted as $F_{\alpha}(\beta)$ in [19]. Also, the usage of osc $(\cdot, \cdot)$ in Theorem 1.7 is different as it counts the oscillations between finite subsets of $\omega_{1}-$ see [19]. 
This paper is organized as follows. Section 2 provides some background on the method of minimal walks and introduces the lower trace function which is used in the statement of the main theorem on oscillations. Section 3 provides a review of elementary submodels. The main combinatorial results of the paper are proved in Section 4. Theorem 1.1 is deduced in Section 5 and a two-place function $o:\left[\omega_{1}\right]^{2} \rightarrow \mathbb{T}$ is introduced which harnesses most of the strength of the more technical main theorem. Theorem 1.2 is then deduced in Section 6 after some motivation is provided. The paper closes with Section 7 where Theorems 1.3 and 1.4 are proved. This final section also contains a basic analysis of the space in Theorem 1.3 .

This paper is intended to be accessible to any interested reader who is fluent in set theory. Basic background can be found in, e.g., [10. Elementary submodels will be employed at a number of points in the argument. Ironically, this represents the only non-elementary technique used in the proofs. The essentials are reviewed in Section 3. The reader is referred to III.1 of [6] for more information on elementary submodels and stationary sets. The proofs will also employ the method of minimal walks introduced in [19. The necessary background is presented in Section 2. The reader is referred to 24 for further reading on minimal walks 3

The notation is fairly standard. All ordinals are von Neumann ordinals - they are the sets consisting of their predecessors. In particular, $n=\{0,1, \ldots, n-1\}$ and the first infinite ordinal $\omega$ is the set of all finite ordinals $\{0,1,2, \ldots\}$ and is identified with the natural numbers. All counting starts at 0 . We will also need the unit circle viewed as the set

$$
\mathbb{T}=\{z \in \mathbb{C}:|z|=1\}
$$

equipped with the usual multiplication operation. If $k$ is a natural number and $X$ is a set, then $[X]^{k}$ is the set of all $k$-element subsets of $X$. If $X$ has a canonical linear ordering associated with it and $a$ is in $[X]^{k}$, then $a$ will be identified with the increasing sequence which enumerates it. If $a$ and $b$ are finite subsets of $\omega_{1}$, then $a<b$ will be used to abbreviate " $\alpha<\beta$ whenever $\alpha$ is in $a$ and $\beta$ is in $b$." Similarly one defines statements such as $\alpha<b$ and $a<\beta$ if $\alpha$ and $\beta$ are ordinals.

\section{THE TRACE FUnCTIONS}

In this section I will provide the necessary background on minimal walks; see 24 for further reading. With the exception of the definition and axiomatization of $L$, everything discussed in this section is contained either explicitly or implicitly in [19. Minimal walks are facilitated by a $C$-sequence which one uses to "walk" from an ordinal $\beta$ down to a smaller ordinal $\alpha$.

Definition 2.1. A $C$-sequence is a sequence $\left\langle C_{\alpha}: \alpha<\omega_{1}\right\rangle$ such that $C_{\alpha}$ is a cofinal subset of $\alpha$ and if $\gamma<\alpha$, then $C_{\alpha} \cap \gamma$ is finite. It will be useful at certain points to assume that 0 is an element of every $C_{\alpha}$.

The following two functions will be of interest to us. The upper trace will not be necessary, but is useful in making the other definitions more transparent.

${ }^{3}$ 24] is an updated, expanded, and clearer account of the methods and results of [19]. My references to [19] are for historical accuracy. 
Definition 2.2 (24] (upper trace)). If $\alpha \leq \beta$, then $\operatorname{Tr}(\alpha, \beta)$ is defined recursively by

$$
\begin{gathered}
\operatorname{Tr}(\alpha, \alpha)=\emptyset, \\
\operatorname{Tr}(\alpha, \beta)=\operatorname{Tr}\left(\alpha, \min \left(C_{\beta} \backslash \alpha\right)\right) \cup\{\beta\} .
\end{gathered}
$$

Definition 2.3 (lower trace). If $\alpha \leq \beta$, then $L(\alpha, \beta)$ is defined recursively by

$$
\begin{gathered}
L(\alpha, \alpha)=\emptyset, \\
L(\alpha, \beta)=L\left(\alpha, \min \left(C_{\beta} \backslash \alpha\right)\right) \cup\left\{\max \left(C_{\beta} \cap \alpha\right)\right\} \backslash \max \left(C_{\beta} \cap \alpha\right) .
\end{gathered}
$$

Hence the upper trace $\operatorname{Tr}(\alpha, \beta)$ is enumerated by the sequence $\beta=\beta_{0}>\beta_{1}>$ $\cdots>\beta_{l-1}$ where $\beta_{i+1}=\min \left(C_{\beta_{i}} \backslash \alpha\right)$ and $\alpha=\beta_{l}$ is in $C_{\beta_{l-1}}$. It is easily verified that the lower trace $L(\alpha, \beta)$ is listed as $\xi_{0} \leq \xi_{1} \leq \cdots \leq \xi_{l-1}$, where

$$
\xi_{k}=\max \bigcup_{j=0}^{k}\left(C_{\beta_{j}} \cap \alpha\right) .
$$

While the upper trace $\operatorname{Tr}(\alpha, \beta)$ and the full lower trace $F(\alpha, \beta)$ of a walk are well studied (see [24]), this is, to my knowledge, the first explicit analysis of the lower trace $L(\alpha, \beta) \subseteq F(\alpha, \beta)$.

For our purposes, the lower trace can be axiomatized by the following facts.

Fact 1. If $\alpha \leq \beta \leq \gamma$ and $L(\beta, \gamma)<L(\alpha, \beta)$, then

$$
L(\alpha, \gamma)=L(\alpha, \beta) \cup L(\beta, \gamma) .
$$

Proof. Let $\alpha, \beta$, and $\gamma$ satisfy the hypothesis of the fact. Observe that $L(\beta, \gamma)<\alpha$ and hence $C_{\zeta} \cap \alpha=C_{\zeta} \cap \beta$ whenever $\zeta$ is in $\operatorname{Tr}(\beta, \gamma)$. Hence $\beta$ is in $\operatorname{Tr}(\alpha, \gamma)$ and $\operatorname{Tr}(\alpha, \gamma)=\operatorname{Tr}(\alpha, \beta) \cup \operatorname{Tr}(\beta, \gamma)$. Let

$$
\gamma=\gamma_{0}>\gamma_{1}>\ldots>\gamma_{l-1}
$$

enumerate $\operatorname{Tr}(\alpha, \gamma)$ and let $l_{0}$ be such that $\beta=\gamma_{l_{0}}$. Then $L(\alpha, \gamma)$ is listed as

$$
\xi_{0} \leq \xi_{1} \leq \cdots \leq \xi_{l-1},
$$

where

$$
\xi_{k}=\max \bigcup_{j=0}^{k}\left(C_{\gamma_{j}} \cap \alpha\right) .
$$

If $k<l_{0}$, then $C_{\gamma_{k}} \cap \alpha=C_{\gamma_{k}} \cap \beta$ and so $L(\beta, \gamma)=\left\{\xi_{k}\right\}_{k=0}^{l-1}$. On the other hand,

$$
\max \left(C_{\gamma_{l_{0}}} \cap \alpha\right)>\xi_{l_{0}-1}
$$

and so if $k \geq l_{0}$,

$$
\max \bigcup_{j=0}^{k}\left(C_{\gamma_{j}} \cap \alpha\right)=\max \bigcup_{j=l_{0}}^{k}\left(C_{\gamma_{j}} \cap \alpha\right)
$$

and hence $L(\alpha, \beta)=\left\{\xi_{k}\right\}_{k=l_{0}}^{l-1}$.

Fact 2. If $\alpha<\beta$, then $L(\alpha, \beta)$ is a non-empty finite set, and if $0<\beta$ is a limit ordinal, then

$$
\lim _{\alpha \rightarrow \beta} \min L(\alpha, \beta)=\beta
$$


Proof. This follows immediately from the observation that

$$
\min L(\alpha, \beta)=\max \left(C_{\beta} \cap \alpha\right) .
$$

Remark 2.4. Notice that if $0<\beta<\gamma$ are fixed and $\beta$ is a limit ordinal, then Fact 2 implies that for all but a bounded set of $\alpha<\beta$, the inequality in Fact 1 holds.

At this point I recall the following definition.

Definition 2.5. If $\left\langle e_{\beta}: \beta<\omega_{1}\right\rangle$ is a sequence of functions such that $e_{\beta}: \beta \rightarrow \omega$ for all $\beta<\omega_{1}$, then the sequence is coherent if whenever $\beta \leq \beta^{\prime}<\omega_{1}, e_{\beta^{\prime}}\lceil\beta$ differs from $e_{\beta}$ only on a finite set.

For brevity, if I say that $\left\langle e_{\beta}: \beta<\omega_{1}\right\rangle$ is a coherent sequence of finite-toone functions, then it is implicit that each $e_{\beta}$ is a function from $\beta$ to $\omega$. The main combinatorial result of the paper will be concerned with counting oscillations between pairs of elements of such sequences. The following construction provides a standard example.

Definition 2.6 ([19] (maximal weight)). If $\alpha \leq \beta$, then $\varrho_{1}(\alpha, \beta)$ is defined recursively by

$$
\begin{gathered}
\varrho_{1}(\alpha, \alpha)=0, \\
\varrho_{1}(\alpha, \beta)=\max \left(\left|C_{\beta} \cap \alpha\right|, \varrho_{1}\left(\alpha, \min \left(C_{\beta} \backslash \alpha\right)\right)\right) .
\end{gathered}
$$

Alternately, $\varrho_{1}(\alpha, \beta)$ is the maximum value of the form $\left|C_{\zeta} \cap \alpha\right|$ where $\zeta$ ranges over $\operatorname{Tr}(\alpha, \beta)$. For each $\beta<\omega_{1}$, define $e_{\beta}: \beta \rightarrow \omega$ by $e_{\beta}(\alpha)=\varrho_{1}(\alpha, \beta)$.

Fact $3([19]) \cdot\left\langle e_{\beta}: \beta<\omega_{1}\right\rangle$ is a coherent sequence of finite-to-one functions.

Proof. Let $\beta \leq \beta^{\prime}<\omega_{1}$ and $n<\omega$ be given and set $D$ equal to the set of all $\alpha<\beta$ such that either $e_{\beta}(\alpha) \leq n$ or $e_{\beta}(\alpha) \neq e_{\beta^{\prime}}(\alpha)$. It suffices to show that $D$ has no limit points.

To this end, suppose $\delta \leq \beta$. It is easy to check that there is a $\delta_{0}<\delta$ such that

$$
\begin{gathered}
\operatorname{Tr}(\alpha, \beta)=\operatorname{Tr}(\alpha, \delta) \cup \operatorname{Tr}(\delta, \beta), \\
\operatorname{Tr}\left(\alpha, \beta^{\prime}\right)=\operatorname{Tr}(\alpha, \delta) \cup \operatorname{Tr}\left(\delta, \beta^{\prime}\right), \\
\left|C_{\delta} \cap \alpha\right|>n
\end{gathered}
$$

whenever $\delta_{0}<\alpha<\delta$. Notice that if $\delta$ is a successor, then this can be made vacuously true by letting $\delta_{0}=\delta-1$. If $\delta_{0}<\alpha<\delta$, then

$$
\begin{gathered}
e_{\beta}(\alpha) \geq\left|C_{\delta} \cap \alpha\right|>n, \\
e_{\beta}(\alpha)=e_{\delta}(\alpha)=e_{\beta^{\prime}}(\delta)
\end{gathered}
$$

and hence $\alpha$ is not in $D$. Consequently $\delta$ is not a limit point of $D$.

Remark 2.7. It is interesting to note that while coherence and the finite-to-one property are at tension with each other, the verifications of these properties in the previous fact are virtually identical.

Remark 2.8. It follows from Fact 3 that $T\left(\varrho_{1}\right)=\left\{e_{\beta}\left\lceil\alpha: \alpha \leq \beta<\omega_{1}\right\}\right.$ is an Aronszajn tree - its levels and chains are countable.

Throughout this paper we will assume that $L$ and $\left\langle e_{\beta}: \beta<\omega_{1}\right\rangle$ satisfy Facts 1]3. We will also need the following two-place function. 
Definition 2.9. If $\alpha<\beta<\omega_{1}$, then let $\Delta(\alpha, \beta)$ be the least ordinal $\xi$ such that either $e_{\alpha}(\xi) \neq e_{\beta}(\xi)$ or $\xi=\alpha$.

\section{BASIC FACTS ABOUT ELEMENTARY SUBMODELS}

In the proof of the main theorem, we will need the following facts about elementary submodels of $H\left(\aleph_{1}^{+}\right)$. Recall that $H\left(\aleph_{1}^{+}\right)$is the collection of all sets of hereditary cardinality at most $\aleph_{1}$. This structure is of interest to us since it contains all of the objects relevant to us (e.g. $\omega_{1}, L$, and $\left\langle e_{\beta}: \beta<\omega_{1}\right\rangle$ ), is closed under taking subsets, and satisfies all of the axioms of ZFC except the power set axiom. An elementary submodel $M$ of $H\left(\aleph_{1}^{+}\right)$is a subset of $H\left(\aleph_{1}^{+}\right)$such that whenever $\phi$ is a logical formula with parameters in $M, M$ satisfies $\phi$ if and only if $H\left(\aleph_{1}^{+}\right)$satisfies $\phi$. The following fact is well known.

Fact 4. If $M$ is a countable elementary submodel of $H\left(\aleph_{1}^{+}\right)$, then $M \cap \omega_{1}$ is an ordinal. Moreover, if $F$ is a countable subset of $H\left(\aleph_{1}^{+}\right)$, then the set of all ordinals of the form $M \cap \omega_{1}$ such that $M$ is a countable elementary submodel of $H\left(\aleph_{1}^{+}\right)$with $F \subseteq M$ contains a club 4

The following standard facts are very useful when working with countable elementary submodels.

Fact 5. If $M$ is a countable elementary submodel of $H\left(\aleph_{1}^{+}\right)$which contains some element $X$, then $X$ is countable iff $X \subseteq M$.

Fact 6. If $M$ is a countable elementary submodel of $H\left(\aleph_{1}^{+}\right)$which contains as an element some subset $A$ of $\omega_{1}$, then $A$ is uncountable iff $A \cap M \cap \omega_{1}$ is unbounded in $M \cap \omega_{1}$.

Fact 7. If $M$ is a countable elementary submodel of $H\left(\aleph_{1}^{+}\right), X$ is in $H\left(\aleph_{1}^{+}\right)$and $X$ is definable from a logical formula with parameters in $M$, then $X$ is in $M$.

Remark 3.1. The above facts remain true if $\aleph_{1}^{+}$is replaced with any uncountable regular cardinal.

\section{Oscillations on the lOWER trace}

In this section I will prove the main combinatorial theorem of the paper. We will take the following as our definition of the oscillation function.

Definition 4.1. Suppose that $s$ and $t$ are two functions defined on a common finite set of ordinals $F$. Let $\operatorname{Osc}(s, t ; F)$ be the set of all $\xi$ in $F \backslash\{\min F\}$ such that $s\left(\xi^{-}\right) \leq t\left(\xi^{-}\right)$and $s(\xi)>t(\xi)$ where $\xi^{-}$is the greatest element of $F$ less than $\xi$ 5

The following notation will be convenient.

Definition 4.2. If $\alpha<\beta<\omega_{1}$, let $\operatorname{Osc}(\alpha, \beta)$ denote

$$
\operatorname{Osc}\left(e_{\alpha}, e_{\beta} ; L(\alpha, \beta)\right)
$$

and $\operatorname{osc}(\alpha, \beta)$ denote the cardinality of $\operatorname{Osc}(\alpha, \beta)$.

\footnotetext{
${ }^{4} \mathrm{~A}$ subset of $\omega_{1}$ is a $c l u b$ if it is closed and unbounded.

${ }^{5}$ Actually, we will later see that we are really interested in counting oscillations between the relations $=$ and $\neq$ (rather than the more conventional $<$ and $>$ ). The above definition is a compromise between the two.
} 
Theorem 4.3. For every $\mathscr{A} \subseteq\left[\omega_{1}\right]^{k}$ and $\mathscr{B} \subseteq\left[\omega_{1}\right]^{l}$ which are uncountable families of pairwise disjoint sets and every $n<\omega$, there are a in $\mathscr{A}$ and $b_{m}(m<n)$ in $\mathscr{B}$ such that for all $i<k, j<l$, and $m<n$ :

$$
\begin{aligned}
a & <b_{m}, \\
\operatorname{osc}\left(a(i), b_{m}(j)\right) & =\operatorname{osc}\left(a(i), b_{0}(j)\right)+m .
\end{aligned}
$$

Theorem 4.3 will be derived from the following lemma.

Lemma 4.4. Let $\mathscr{A} \subseteq\left[\omega_{1}\right]^{k}$ and $\mathscr{B} \subseteq\left[\omega_{1}\right]^{l}$ be uncountable and pairwise disjoint. There is a closed and unbounded set of $\delta<\omega_{1}$ such that if $a$ is in $\mathscr{A} \backslash \delta, b$ is in $\mathscr{B} \backslash \delta$, and $R$ is in $\{=>>\}$, then there are $a^{+}$in $\mathscr{A} \backslash \delta$, and $b^{+}$in $\mathscr{B} \backslash \delta$ such that for all $i<k$ and $j<l$ 6

(i) $\max L(\delta, b(j))$ is less than both $\Delta\left(a(i), a^{+}(i)\right)$ and $\Delta\left(b(j), b^{+}(j)\right)$;

(ii) $L(\delta, b(j))$ is a proper initial part of $L\left(\delta, b^{+}(j)\right)$;

(iii) if $\xi$ is in $L^{+}=L\left(\delta, b^{+}(j)\right) \backslash L(\delta, b(j))$, then $e_{a^{+}(i)}(\xi) R e_{b^{+}(j)}(\xi)$.

Proof. Let $M$ be a countable elementary submodel of $H\left(\aleph_{1}^{+}\right)$which contains everything relevant and let $\delta=M \cap \omega_{1}$. By Fact 4 , it suffices to show that $\delta$ satisfies the conclusion of the theorem.

First suppose that $R$ is $=$. Applying Fact 3 find a $\gamma_{0}<\delta$ satisfying the following conditions:

(1) if $j<l$, then $L(\delta, b(j))<\gamma_{0}$;

(2) if $i<k, j<l$, and $\gamma_{0}<\xi<\delta$, then $e_{a(i)}(\xi)=e_{b(j)}(\xi)$.

Applying Fact 2. pick a $\gamma<\delta$ such that if $\gamma<\xi<\delta$, then $\gamma_{0}<L(\xi, \delta)$. Consider the set $D \subseteq \omega_{1}$ consisting of all $\delta^{+}$such that for some $a^{+}$in $\mathscr{A} \backslash \delta^{+}$and $b^{+}$in $\mathscr{B} \backslash \delta^{+}$the following conditions are satisfied:

(3) $e_{a^{+}(i)}\left\lceil\gamma_{0}=e_{a(i)}\left\lceil\gamma_{0}\right.\right.$ and $e_{b^{+}(j)}\left\lceil\gamma_{0}=e_{b(j)}\left\lceil\gamma_{0}\right.\right.$ for all $i<k$ and $j<l$;

(4) $L\left(\delta^{+}, b^{+}(j)\right)=L(\delta, b(j))$ for all $j<l$;

(5) if $\gamma<\xi<\delta^{+}$, then $\gamma_{0}<L\left(\xi, \delta^{+}\right)$;

(6) if $\gamma_{0}<\xi<\delta^{+}, i<k$, and $j<l$, then $e_{a^{+}(i)}(\xi)=e_{b^{+}(j)}(\xi)$.

Observe that for all $\beta \geq \gamma_{0}, e_{\beta}\left\lceil\gamma_{0}\right.$ is in $M$ since by Fact 3 it differs from $e_{\gamma_{0}}$ on a finite set. Hence $D$ is definable from the parameters $\gamma_{0}, \gamma, e_{a(i)}\left\lceil\gamma_{0}\right.$, etc., which are all elements of $M$. Therefore $D$ is in $M$ by Fact 7 Since $D$ has $\delta$ as a member, it is uncountable by Fact 5 . Hence there is a $\delta^{+}>\delta$ in $D$. Let $a^{+} \in \mathscr{A} \backslash \delta^{+}$and $b^{+} \in \mathscr{B} \backslash \delta^{+}$witness that $\delta^{+}$is in $D$.

Now let $i<k$ and $j<l$ be arbitrary. First observe that

$$
\begin{aligned}
& \gamma_{0} \leq \Delta\left(a(i), a^{+}(i)\right), \\
& \gamma_{0} \leq \Delta\left(b(j), b^{+}(j)\right) .
\end{aligned}
$$

Put $L^{+}=L\left(\delta, \delta^{+}\right)$. Notice that $L(\delta, b(j))=L\left(\delta^{+}, b^{+}(j)\right)<L^{+}$and hence

$$
L\left(\delta, b^{+}(j)\right)=L\left(\delta^{+}, b^{+}(j)\right) \cup L^{+}=L(\delta, b(j)) \cup L^{+}
$$

holds by Fact 1. Since $\delta<\delta^{+}, L^{+}$is non-empty. If $\xi$ is in $L^{+}$, then $\gamma_{0}<\xi<\delta^{+}$ and so

$$
e_{a^{+}(i)}(\xi) R e_{b^{+}(j)}(\xi)
$$

\footnotetext{
${ }^{6}$ It will be convenient to let $\mathscr{A} \backslash \delta$ denote the set of all $a$ in $\mathscr{A}$ such that $\delta \leq a$ whenever $\mathscr{A}$ is a collection of finite subsets of $\omega_{1}$ and $\delta<\omega_{1}$.
} 
Now suppose that $R$ is $>$. Put $E$ equal to the set of all limits $\nu<\omega_{1}$ such that for all $a_{0}$ in $\mathscr{A} \backslash \nu$, all $\nu_{0}<\nu, \varepsilon<\omega_{1}, n<\omega$, and finite $L^{+} \subseteq \omega_{1} \backslash \nu$ there is an $a_{1}$ in $\mathscr{A} \backslash \varepsilon$ with

$$
\begin{gathered}
\nu_{0} \leq \Delta\left(a_{0}(i), a_{1}(i)\right), \\
e_{a_{1}(i)}(\xi)>n
\end{gathered}
$$

whenever $i<k$ and $\xi$ is in $L^{+}$. By Fact $7, E$ is an element of $M$.

Claim 4.5. $\delta$ is in E. In particular, $E$ is uncountable by Fact 5 .

Proof. Let $a_{0}, \nu_{0}, \varepsilon, n$, and $L^{+}$be given as in the definition of $E$ for $\nu=\delta$. By Fact 3 we may assume without loss of generality that $\nu_{0}$ is an upper bound for all $\xi<\delta$ such that $e_{a_{0}(i)}(\xi) \leq n$ for some $i<k$. Now, applying the elementarity of $M$, there is a $\delta^{+}$above $\varepsilon, \delta$, and $\max L^{+}$and an $a_{1}$ in $\mathscr{A} \backslash \delta^{+}$such that the following conditions are satisfied:

(7) for all $i<k, e_{a_{0}(i)}\left\lceil\nu_{0}=e_{a_{1}(i)}\left\lceil\nu_{0}\right.\right.$;

(8) if $\nu_{0}<\xi<\delta^{+}$and $i<k$, then $e_{a_{1}(i)}(\xi)>n$.

Since $L^{+} \subseteq \delta^{+} \backslash \delta$, this completes the proof of the claim.

Applying the elementarity of $M$ and the uncountability of $E$, find an element $\gamma_{0}$ of $E$ such that $L(\delta, b(j))<\gamma_{0}<\delta$ for all $j<l$. Applying Fact 2, find a $\gamma<\delta$ such that if $\gamma<\xi<\delta$, then $\gamma_{0}<L(\xi, \delta)$. Again using the elementarity of $M$, select a limit $\delta^{+}>\delta$ and a $b^{+}$in $\mathscr{B} \backslash \delta^{+}$so that the following conditions are satisfied:

(9) $e_{b^{+}(j)}\left\lceil\gamma_{0}=e_{b(j)}\left\lceil\gamma_{0}\right.\right.$ for all $j<l$;

(10) $L\left(\delta^{+}, b^{+}(j)\right)=L(\delta, b(j))$ for all $j<l$;

(11) if $\gamma<\xi<\delta^{+}$, then $\gamma_{0}<L\left(\xi, \delta^{+}\right)$.

Put $L^{+}=L\left(\delta, \delta^{+}\right)$. Applying the definition of $E$, find an $a^{+}$in $\mathscr{A} \backslash \delta$ such that for all $i<k, j<l$, and $\xi$ in $L^{+}$,

$$
\begin{gathered}
L(\delta, b(j))<\Delta\left(a(i), a^{+}(i)\right), \\
e_{a^{+}(i)}(\xi)>e_{b^{+}(j)}(\xi) .
\end{gathered}
$$

The rest of the verification is as in the previous case. This completes the proof of Lemma 4.4.

Now we are ready to prove Theorem 4.3 .

Proof. Let $\mathscr{A}$ and $\mathscr{B}$ be given and select a countable elementary submodel $M$ of $H\left(\aleph_{1}^{+}\right)$containing everything relevant, setting $\delta=M \cap \omega_{1}$. Since $M$ contains $\mathscr{A}$ and $\mathscr{B}$, the closed and unbounded set provided by Lemma4.4 is in $M$ and therefore $\delta$ is in this closed and unbounded set. Using Lemma 4.4, it is possible to select $a_{m}$ $(m<\omega)$ in $\mathscr{A} \backslash \delta, b_{m}(m<\omega)$ in $\mathscr{B} \backslash \delta$, and $\xi_{m}(m<\omega)$ in $\delta$ so that for all $m<\omega$ the following conditions are satisfied whenever $i<k$ and $j<l$ :

(12) $L\left(\delta, b_{m}(j)\right)$ is a proper initial part of $L\left(\delta, b_{m+1}(j)\right)$;

(13) $L\left(\delta, b_{m+1}(j)\right) \backslash L\left(\delta, b_{m}(j)\right)$ does not depend on $j$ and contains $\xi_{m}$ as an element;

(14) Osc $\left(a_{m+1}(i), b_{m+1}(j) ; L\left(\delta, b_{m+1}(j)\right)\right)$ is formed by adding $\xi_{m}$ to Osc $\left(a_{m}(i), b_{m}(j) ; L\left(\delta, b_{m}(j)\right)\right)$;

(15) if $m^{\prime}<m$, then $\xi_{m^{\prime}}$ is strictly less than both $\Delta\left(a_{m}(i), a_{m+1}(i)\right)$ and $\Delta\left(b_{m}(j), b_{m+1}(j)\right)$; 
(16) $e_{a_{m}(i)}\left(\max L\left(\delta, b_{m}(j)\right)\right)>e_{b_{m}(j)}\left(\max L\left(\delta, b_{m}(j)\right)\right)$.

Notice that, once $a_{m}$ and $b_{m}$ have been selected, Lemma 4.4 is applied twice in order to obtain $a_{m+1}$ and $b_{m+1}$ - first with $R$ being $=$ and then with $R$ being $>$. The ordinal $\xi_{m}$ is the minimum of what might be described as

$$
L\left(\delta, b_{m+1}(j)\right) \backslash L\left(\delta, b_{m+1 / 2}(j)\right) .
$$

Now let $n$ be given. Pick a $\gamma_{0}<\delta$ which is an upper bound for each $L\left(\delta, b_{n}(j)\right)$ for $j<l$ and all $\xi<\delta$ such that for some $m, m^{\prime} \leq n$ and $j, j^{\prime}<l$,

$$
e_{b_{m}(j)}(\xi) \neq e_{b_{m^{\prime}}\left(j^{\prime}\right)}(\xi)
$$

(the latter set is finite by Fact 3). Using the elementarity of $M$ and Fact 2, select an $a$ in $\mathscr{A}$ such that $a<\delta$ and for all $i<k$ and $j<l$,

$$
\begin{gathered}
L\left(\delta, b_{n}(j)\right)<\Delta\left(a(i), a_{n}(i)\right), \\
\gamma_{0}<L(a(i), \delta) .
\end{gathered}
$$

Now let $i<k, j<l$, and $m<n$ be fixed. It follows from Fact 1 that

$$
L\left(a(i), b_{m}(j)\right)=L(a(i), \delta) \cup L\left(\delta, b_{m}(j)\right) .
$$

Finally, $e_{b_{m}(j)} \uparrow L(a(i), \delta)$ does not depend on $m$ and therefore

$$
\text { Osc }\left(a(i), b_{0}(j) ; L(a(i), \delta)\right)=\operatorname{Osc}\left(a(i), b_{m}(j) ; L(a(i), \delta)\right) .
$$

By (16), Osc $\left(a(i), b_{m}(j)\right)$ is the union

$$
\text { Osc }\left(a(i), b_{m}(j) ; L(a(i), \delta)\right) \cup \operatorname{Osc}\left(a(i), b_{m}(j) ; L\left(\delta, b_{m}(j)\right)\right)
$$

(i.e. no new oscillation occurs at the "seam"). Hence, by (14),

$$
\operatorname{Osc}\left(a(i), b_{m}(j)\right)=\operatorname{Osc}\left(a(i), b_{0}(j)\right) \cup\left\{\xi_{m^{\prime}}: m^{\prime}<m\right\} .
$$

This completes the proof of Theorem 4.3

\section{A Negative PARTition RELATiON AND THE COLORING $O$}

We will now consider the following function.

Definition 5.1. If $\alpha<\beta$, define

$$
o(\alpha, \beta)=z_{\alpha}^{\mathrm{osc}(\alpha, \beta)+1} .
$$

This definition is motivated by the following theorem - commonly known as Kronecker's Theorem - discovered independently by Kronecker and Tchebychef (see, e.g., [1]).

Kronecker's Theorem ([8], 17]). Suppose that $z_{i}(i<k)$ are elements of $\mathbb{T}$ which are rationally independent. For every $\varepsilon>0$ there is a natural number $n_{\varepsilon}$ such that if $u, v$ are in $\mathbb{T}^{k}$, there is an $m<n_{\varepsilon}$ such that for all $i<k$,

$$
\left|u_{i} z_{i}^{m}-v_{i}\right|<\varepsilon \text {. }
$$

Remark 5.2. In the usual formulation of this theorem, $n_{\varepsilon}$ depends on $u$ and $v$. The uniformity of $n_{\varepsilon}$ as $u$ and $v$ vary follows from a standard compactness argument.

For the remainder of the paper, fix a sequence $\left\langle z_{\alpha}: \alpha<\omega_{1}\right\rangle$ of elements of $\mathbb{T}$ which are rationally independent. This is possible since, given any countable rationally independent subset $I$ of $\mathbb{T}$, there are only countably many $z$ for which $I \cup\{z\}$ is rationally dependent.

The following function will also be useful. 
Definition 5.3. The function $*: \mathbb{T} \rightarrow \omega$ is defined by letting $*(z)=n$ iff there is a $\theta$ in $\left(\frac{2 \pi}{n+2}, \frac{2 \pi}{n+1}\right]$ such that $z=e^{i \theta}$. If $f$ is a function taking values in $\mathbb{T}$, then $f^{*}$ will denote the composition of $f$ followed by $*$.

The point is that $*$ takes all values on every dense subset of $\mathbb{T}$. The notation for $*$ is chosen to mimic its usage in 24. We will shortly see that $o^{*}$ defines a partition of $\left[\omega_{1}\right]^{2}$ with strong properties.

In [18, Todorcevic considered two variations of $\omega_{1} \rightarrow\left(\omega_{1} ; \omega_{1}\right)_{2}^{2}$ which are closely related to $(\mathrm{S})$ and $(\mathrm{L})$ respectively:

$$
\begin{aligned}
& \omega_{1} \rightarrow\left(\omega_{1},\left(\omega_{1} ; \text { fin } \omega_{1}\right)\right)^{2}, \\
& \omega_{1} \rightarrow\left(\omega_{1},\left(\text { fin } \omega_{1} ; \omega_{1}\right)\right)^{2} .
\end{aligned}
$$

The statement $\omega_{1} \rightarrow\left(\omega_{1},\left(\omega_{1} ; \text { fin } \omega_{1}\right)\right)^{2}$ asserts that if $c:\left[\omega_{1}\right]^{2} \rightarrow 2$, then either

(A) there is an uncountable $X \subseteq \omega_{1}$ such that $c$ is constantly 0 on $[X]^{2}$ or

(B) there are uncountable $A \subseteq \omega_{1}$ and uncountable pairwise disjoint $\mathscr{B} \subseteq$ $\left[\omega_{1}\right]^{<\aleph_{0}}$ such that for all $\alpha$ in $A$ and $b$ in $\mathscr{B}$ with $\alpha<b$, there is a $\beta$ in $b$ with $c(\alpha, \beta)=1$.

The statement $\omega_{1} \rightarrow\left(\omega_{1},\left(\text { fin } \omega_{1} ; \omega_{1}\right)\right)^{2}$ is similarly defined by replacing $A \subseteq \omega_{1}$ by a pairwise disjoint $\mathscr{A} \subseteq\left[\omega_{1}\right]^{<\aleph_{0}}$ and $\mathscr{B}$ by $B \subseteq \omega_{1}$. These imply (S) and (L) respectively (see [18]) and are each immediate consequences of $\omega_{1} \rightarrow\left(\omega_{1} ;\left(\omega_{1} ; \omega_{1}\right)\right)_{2}^{2}$.

In [18], Todorcevic showed that $\omega_{1} \rightarrow\left(\omega_{1},\left(\omega_{1} ; \text { fin } \omega_{1}\right)\right)^{2}$ was relatively consistent with ZFC and in fact follows from PFA. We will now see that $o^{*}$ witnesses a strong failure of $\omega_{1} \rightarrow\left(\omega_{1}\right.$, (fin $\left.\left.\omega_{1} ; \omega_{1}\right)\right)^{2}$.

Theorem 5.4. Let $\mathscr{A} \subseteq\left[\omega_{1}\right]^{k}$ and $\mathscr{B} \subseteq\left[\omega_{1}\right]^{l}$ be uncountable families of pairwise disjoint sets. For every $\chi: k \rightarrow \omega$ and $\phi: k \rightarrow l$ there are $a$ in $\mathscr{A}$ and $b$ in $\mathscr{B}$ such that $a<b$ and for all $i<k$,

$$
o^{*}(a(i), b(\phi(i)))=\chi(i) .
$$

By a "stretching" argument which can be found at the beginning of Section 4 of [19], Theorem 5.4 yields the following corollary.

Corollary 5.5. $\omega_{1} \nrightarrow\left[\omega_{1} ; \omega_{1}\right]_{\omega_{1}}^{2}$.

Theorem 5.4 is an immediate consequence of the following theorem.

Theorem 5.6. Let $\mathscr{A} \subseteq\left[\omega_{1}\right]^{k}$ and $\mathscr{B} \subseteq\left[\omega_{1}\right]^{l}$ be uncountable families of pairwise disjoint sets. For every sequence $U_{i}(i<k)$ of open neighborhoods in $\mathbb{T}$ and every $\phi: k \rightarrow l$ there are $a$ in $\mathscr{A}$ and $b$ in $\mathscr{B}$ such that $a<b$ and for all $i<k$,

$$
o(a(i), b(\phi(i))) \in U_{i} .
$$

Proof. Without loss of generality, we may assume that each $U_{i}$ is an $\varepsilon$-ball about a point $v_{i}$ for some fixed $\varepsilon>0$. By refining $\mathscr{A}$ if necessary, we may assume that the $n_{\varepsilon}$ given by Kronecker's theorem for the sequence $z_{a(i)}(i<k)$ are uniform for $a$ in $\mathscr{A}$. Let $a$ in $\mathscr{A}$ and $b_{m}\left(m<n_{\varepsilon}\right)$ in $\mathscr{B}$ be such that for all $i<k, j<l$, and $m<n_{\varepsilon}$,

$$
\begin{gathered}
a<b_{m}, \\
\operatorname{osc}\left(a(i), b_{m}(j)\right)=\operatorname{osc}\left(a(i), b_{0}(j)\right)+m .
\end{gathered}
$$


For each $i<k$, put $u_{i}=o\left(a(i), b_{0}(\phi(i))\right)$. By choice of $n_{\varepsilon}$, there is an $m<n_{\varepsilon}$ such that for all $i<k$,

$$
\left|u_{i} z_{a(i)}^{m}-v_{i}\right|<\varepsilon
$$

or, equivalently, $o\left(a(i), b_{m}(\phi(i))\right)$ is in $U_{i}$. This finishes the proof.

Question 5.7 (PFA $\sqrt{7})$. If $c:\left[\omega_{1}\right]^{2} \rightarrow 2$, are there $A \subseteq \omega_{1}$ and $\mathscr{B} \subseteq\left[\omega_{1}\right]^{2}$ which are uncountable with $\mathscr{B}$ being pairwise disjoint and a $\chi: 2 \rightarrow 2$ such that for all $\alpha \in A$ and $b \in \mathscr{B}$ with $\alpha<b$ there is an $i<2$ such that $c(\alpha, b(i)) \neq \chi(i)$ ?

\section{A FAMILY OF BINARY RELATIONS ON $\omega_{1}$}

In this section we will consider the family of binary relations on $\omega_{1}$ with the Tukey order.

Definition 6.1 (25] Tukey order). If $R$ and $S$ are binary relations, then we write $R \leq S$ iff there are functions $f: \operatorname{dom}(R) \rightarrow \operatorname{dom}(S)$ and $g: \operatorname{ran}(S) \rightarrow \operatorname{ran}(R)$ such that

$$
f(x) S y \text { implies } x R g(y) \text {. }
$$

This order was first considered by Tukey in the class of transitive relations [25]. It makes sense, however, to consider this order in the more general setting of the class of binary relations (see [26]).

Our focus will be on binary relations on $\omega_{1}$. Two fundamental examples are the well order $\omega_{1}$ and the family $\left[\omega_{1}\right]^{<\aleph_{0}}$ of finite subsets of $\omega_{1}$ ordered by inclusion. We will also need a few standard operations on relations. If $R$ and $S$ are relations, then $R \oplus S$ is the relation which is the disjoint union of $R$ and $S$. If $m$ is a cardinal and $R$ is a relation, then we will let $m \cdot R$ denote the direct sum of $m$ copies of $R$. If $R$ and $S$ are two binary relations, then $R \wedge S$ and $R \vee S$ are the relations with domains $\operatorname{dom}(R) \times \operatorname{dom}(S)$ and ranges $\operatorname{ran}(R) \times \operatorname{ran}(S)$ such that

$$
\begin{gathered}
(a, b) R \wedge S(c, d) \text { iff } a R c \text { or } b S d, \\
(a, b) R \vee S(c, d) \text { iff } a R c \text { and } b S d .
\end{gathered}
$$

The "join" $R \vee S$ is often denoted $R \times S$. It is easily verified that $\wedge$ and $\vee$ give lower and upper bounds respectively 8

It was observed that the binary relations on $\omega_{1}$ which one knew how to construct in ZFC were either below $\aleph_{0} \cdot \omega_{1}$ or above $\left[\omega_{1}\right]^{<\aleph_{0}}$ in the Tukey order.

Example 6.2. Suppose that $r_{\alpha}\left(\alpha<\omega_{1}\right)$ is a sequence of distinct reals. Define $\alpha R \beta$ iff $\alpha<\beta$ and $r_{\alpha}<r_{\beta}$.

The relation $R$ is essentially Sierpiński's partition which witnesses $\left.\omega_{1} \not \omega_{1}\right)_{2}^{2}$. In this case $R \leq \aleph_{0} \cdot \omega_{1}$ by the following Tukey maps:

$$
\begin{gathered}
f(\alpha)=\left(\min \left\{n: r_{\alpha}<q_{n}\right\}, \alpha\right), \\
g(\alpha, n)=\min \left\{\beta: \alpha<\beta \wedge q_{n}<r_{\beta}\right\},
\end{gathered}
$$

where $\left\{q_{n}\right\}_{n<\omega}$ is an enumeration the rationals. On the other hand, the following example shows that with an additional hypothesis such as $\diamond$ one can construct more complex relations.

\footnotetext{
${ }^{7}$ Or any appropriate hypothesis; the same comment applies to Conjecture 4 below.

${ }^{8} \mathrm{In}$ general these need not be optimal - they are in the class of directed relations.
} 
Example 6.3. Let $R$ be a tree order on a set $T$. If $R \leq \aleph_{0} \cdot \omega_{1}$, then $T$ is the union of countably many $R$-chains. If $\left[\omega_{1}\right]^{<\aleph_{0}} \leq R$, then $T$ contains an uncountable $R$ antichain. In particular, if $(T, R)$ is a Suslin tree, then $R \not \leq \aleph_{0} \cdot \omega_{1}$ and $\left[\omega_{1}\right]^{<\aleph_{0}} \not \leq R$.

Observations such as these led Todorcevic to make the following conjecture.

Conjecture (PFA). If $R$ is a binary relation, then either $R \leq \aleph_{0} \cdot \omega_{1}$ or else $\left[\omega_{1}\right]^{<\aleph_{0}} \leq R$.

This can be considered a basis conjecture in the following sense. Let $\mathscr{R}$ denote the class of all binary relations $R$ on $\omega_{1}$ such that $R$ is not reducible to $\aleph_{0} \cdot \omega_{1}$. Then the above conjecture is just the assertion that $\mathscr{R}$ has a single element basis consisting of $\left[\omega_{1}\right]^{<\aleph_{0}}$. This conjecture was given further plausibility by the following theorem which implies that it is true for transitive relations.

Theorem $6.4(22](\mathrm{PFA}))$. Every transitive relation on $\omega_{1}$ is Tukey equivalent to one of the following for some non-negative integers $n_{i}(i<5)$ :

(i) $n_{0} \cdot 1 \oplus n_{1} \cdot \omega \oplus n_{2} \cdot \omega_{1} \oplus n_{3} \cdot \omega \times \omega_{1} \oplus n_{4} \cdot\left[\omega_{1}\right]^{<\aleph_{0}}$;

(ii) $\aleph_{0} \cdot 1 \oplus n_{2} \cdot \omega_{1} \oplus n_{3} \cdot \omega \times \omega_{1} \oplus n_{4} \cdot\left[\omega_{1}\right]^{<\aleph_{0}}$;

(iii) $\aleph_{0} \cdot \omega_{1} \oplus n_{4} \cdot\left[\omega_{1}\right]^{<\aleph_{0}}$;

(iv) $\aleph_{0} \cdot\left[\omega_{1}\right]<\aleph_{0}$;

(v) $=$.

We will now see, however, that this conjecture is provably false.

Definition 6.5. Let $R$ denote the relation with domain and range $\omega_{1}$ defined by letting $\alpha R \beta$ iff $\alpha=\beta$ or $\alpha<\beta$ and $o^{*}(\alpha, \beta)>0$. If $X$ is an uncountable subset of $\omega_{1}$, let $R_{X}$ denote the relation with domain $X$ and range $\omega_{1}$ which is the restriction of $R$ to $X \times \omega_{1}$.

It is easily verified that the enumeration map $\varepsilon: \omega_{1} \rightarrow X$ and the identity map on $\omega_{1}$ witness $\omega_{1} \leq R_{X}$ for every uncountable $X$. If $X \subseteq Y$, then the inclusion and identity maps witness $R_{X} \leq R_{Y}$. The following shows that none of these relations is above $\left[\omega_{1}\right]^{<\aleph_{0}}$ in the Tukey order.

Theorem 6.6. $\left[\omega_{1}\right]^{<\aleph_{0}} \not \leq R$.

Proof. Suppose that $f:\left[\omega_{1}\right]^{<\aleph_{0}} \rightarrow \omega_{1}$ and $g: \omega_{1} \rightarrow\left[\omega_{1}\right]^{<\aleph_{0}}$ are functions. It suffices to show that they are not Tukey reductions.

Applying the pressing down lemma, find a stationary $B \subseteq \omega_{1}$ and a finite $x_{0} \subseteq \omega_{1}$ such that $g(\beta) \cap \beta=x_{0}$ for all $\beta$ in $B$. Let $\mathscr{X}=\left\{x \in\left[\omega_{1}\right]^{<\aleph_{0}}: x \nsubseteq x_{0}\right\}$ and $A=\{f(x): x \in \mathscr{X}\}$. By refining $B$ if necessary, we may assume that if $\alpha$ is in $A$ and $\beta$ is in $B$ with $\alpha<\beta$, then there is an $x$ in $\mathscr{X}$ such that $f(x)=\alpha$ and $x \subseteq \beta$.

If $A$ is countable, then we can find an $\alpha$ in $A$ such that

$$
\{x \in \mathscr{X}: f(x)=\alpha\}
$$

is uncountable. It is then easy to find an $x$ in $\mathscr{X}$ such that $x$ is not contained in $g(\alpha)$ but $f(x)=\alpha$ and hence $f(x) R \beta$, witnessing that $f, g$ are not Tukey maps.

If $A$ is uncountable, apply Theorem 5.4 to obtain an $\alpha$ in $A$ and a $\beta$ in $B$ such that $\alpha<\beta$ and $o^{*}(\alpha, \beta)>0$. Now pick an $x$ in $\mathscr{X}$ such that $f(x)=\alpha$ and $x \subseteq \beta$ and observe that $x$ is not contained in $g(\beta)$ and yet $f(x) R \beta$. 
Theorem 6.7. For all finite sets $F$ of uncountable subsets of $\omega_{1}$,

$$
\bigwedge_{X \in F} R_{X} \not \leq \aleph_{0} \cdot \omega_{1}
$$

Proof. By replacing $F$ with a disjoint refinement if necessary, we may assume without loss of generality that $F$ consists of pairwise disjoint sets. It suffices to show that if

$$
f: \prod_{X \in F} X \rightarrow \omega \times \omega_{1} \quad \text { and } \quad g: \omega \times \omega_{1} \rightarrow \prod_{X \in F} \omega_{1},
$$

then $f$ and $g$ are not Tukey maps. For convenience, we will identify elements of $\prod_{X \in F} X$ with their range. Let $S$ denote the relation on $\omega \times \omega_{1}$ given by $\aleph_{0} \cdot \omega_{1}$. Find uncountable pairwise disjoint $\mathscr{A} \subseteq\left[\omega_{1}\right]^{|F|}$ and an $n$ such that:

(17) for each element $a$ of $\mathscr{A}, a \cap X$ is a singleton for all $X$ in $F$;

(18) $f\left\lceil\mathscr{A}\right.$ is an injection into $\{n\} \times \omega_{1}$;

(19) the ordering on $F$ which is induced by an element $a$ of $\mathscr{A}-X$ is the $i^{\text {th }}$-least element of $F$ if $a(X)$ is the $i^{\text {th }}$-least coordinate of $a$ - does not depend on $a$.

This is possible since if $\prod_{X \in F} X$ is partitioned into countably many pieces, then one piece must contain an uncountable pairwise disjoint family. If the $g$-image of $\{n\} \times \omega_{1}$ is countable, then it is not possible for $f$ and $g$ to be Tukey maps; this is a consequence of the following argument in which $\tilde{\mathscr{B}}$ is replaced by the trivial $\Delta$-system with a single element.

Hence we may select an uncountable $\tilde{\mathscr{B}}$ contained in the image of $\{n\} \times \omega_{1}$ under $g$ such that the collection of sets of coordinates of elements of $\tilde{\mathscr{B}}$ forms a $\Delta$-system with root $r$. Let $\mathscr{B}$ be the elements of this $\Delta$-system with $r$ removed and let $k$ denote the uniform cardinality of the members of $\mathscr{B}$. By refining $\tilde{\mathscr{B}}$ if necessary, we may further assume that there is a function $\phi:|F| \rightarrow k \cup\{-1\}$ such that if $X$ is the $i^{\text {th }}$ element in the induced enumeration of $F$ and $\tilde{b}$ is in $\tilde{\mathscr{B}}$, then $\tilde{b}(X)$ is the $\phi(i)^{\text {th }}$-least element of $\operatorname{ran}(\tilde{b}) \backslash r$ if $\tilde{b}(X)$ is not in $r$ and $\phi(i)=-1$ otherwise. Let $\chi$ be constantly 0 on the subset of $|F|$ on which $\phi$ is non-negative.

Applying Theorem 5.4, it is possible to find an $a$ in $\mathscr{A}$, a $\tilde{b}$ in $\tilde{\mathscr{B}}$, and a $\zeta$ with $g(n, \zeta)=\tilde{b}$ such that $r<a<\min (b \backslash r), f(a)$ is $S$-related to $(n, \zeta)$, and for all $X$ in $F$ for which $\tilde{b}(X)$ is not in $r$,

$$
o^{*}(a(X), \tilde{b}(X))=0,
$$

where $b$ denotes the set of coordinates of $\tilde{b}$.

If $X$ is in $F$, then either $\tilde{b}(X)$ is in $r$ in which case $\tilde{b}(X)<a(X)$ or else $\tilde{b}(X)$ is not in $r$ and $c(a(X), \tilde{b}(X))=0$. In either case $a(X)$ is not $R_{X}$-related to $\tilde{b}(X)$. Since this is true for arbitrary $X, a$ is not $\bigwedge_{X \in F} R_{X}$-related to $g(x)$ and yet $f(a) S x$. Consequently $f$ and $g$ are not Tukey maps.

The next theorem shows that the relations $R_{X}\left(X \subseteq \omega_{1}\right)$ are typically incomparable.

Theorem 6.8. If $X \backslash Y$ is uncountable, then $R_{X} \not \leq R_{Y}$.

Proof. Suppose for a contradiction that $f: X \rightarrow Y$ and $g: \omega_{1} \rightarrow \omega_{1}$ are Tukey maps. To obtain a contradiction, it suffices to find an $\alpha$ in $X$ and a $\beta$ in $\omega_{1}$ such that $f(\alpha)<\beta, \alpha<g(\beta), o^{*}(f(\alpha), \beta)=1$, and $o^{*}(\alpha, g(\beta))=0$. Since $f$ and $g$ 
are Tukey reductions it is possible to find an uncountable $X_{0} \subseteq X \backslash Y$ and an uncountable $Z_{0} \subseteq \omega_{1}$ such that $f\left\lceil X_{0}\right.$ and $g\left\lceil Z_{0}\right.$ are injections, the $f$-image of $X_{0}$ is disjoint from $X_{0}$, the $g$-image of $Z_{0}$ is disjoint from $Z_{0}$ unless $g$ is eventually equal to the identity, and the inequalities $\alpha<f(\alpha), \beta=g(\beta), \beta<g(\beta)$ are uniformly true or false as $\alpha$ ranges over $X_{0}$ and $\beta$ ranges over $Z_{0}$. Put

$$
\mathscr{A}=\left\{\{\alpha, f(\alpha)\}: \alpha \in X_{0}\right\}, \quad \mathscr{B}=\left\{\{\beta, g(\beta)\}: \beta \in Z_{0}\right\}
$$

and notice that these families are uncountable and consist of pairwise disjoint sets of uniform cardinality which, in the case of $\mathscr{A}$, is two. By our uniformity assumption on the relations $<$ and $=$, it is possible to find functions $\phi: 2 \rightarrow 2$ and $\chi: 2 \rightarrow 2$ such that:

(20) $\{a(i), b(\phi(i))\}(i<2)$ lists the pairs $\{\alpha, g(\beta)\}$ and $\{f(\alpha), \beta\}$;

(21) $\chi(i)=1$ if $\{a(i), b(\phi(i))\}=\{f(\alpha), \beta\}$ and $\chi(i)=0$ otherwise.

Applying Theorem 5.4 to $\mathscr{A}, \mathscr{B}, \phi$, and $\chi$, there are $a$ in $\mathscr{A}$ and $b$ in $\mathscr{B}$ such that $a<b$ and

$$
o^{*}(a(i), b(\phi(i)))=\chi(i) .
$$

Translating the outcome, it is easily checked that $\alpha \in a \cap X_{0}$ and $\beta \in b \cap Z_{0}$ are as desired.

I will close this section with two questions.

Question 6.9. For which families $\mathscr{F} \subseteq\left[\omega_{1}\right]^{\aleph_{1}}$ is there an $S$ such that $S \not \leq \aleph_{0} \cdot \omega_{1}$ and $S \leq R_{X}$ for all $X$ in $\mathscr{F}$ ?

Question 6.10. Is the collection of all $S$ with $S \not \leq \aleph_{0} \cdot \omega_{1}$ downwards-directed in the Tukey order?

\section{An L SPACE AND the NON-EXISTENCE OF A SMALL BASIS FOR THE REGULAR HAUSDORFF SPACES}

In this section I will give an example of an $L$ space - a regular Hausdorff space which is hereditarily Lindelöf but not hereditarily separable. The question of the existence of such spaces was first asked explicitly in [5], though arguably this question can be traced to Sierpiński's 14, where he constructed Hausdorff examples distinguishing HS and HL, and to Suslin's [15, where he posed his famous hypothesis. For instance, an immediate consequence of Kurepa [11] is that a Suslin line is an example of an L space. From the 1960s until the 1980s, there was a concerted effort to understand both L spaces and their "dual", the S space. I refer the reader to Juhász's [7] and M. E. Rudin's [13] as well as Roitman's more recent [12] for more discussion on these developments. I have selected a few to mention here.

First, Zenor showed that there was a relationship between the existence of certain $\mathrm{S}$ and L spaces.

Theorem 7.1 ([27]). There is a strong $S$ space iff there is a strong L space 9

Hence the difference in the existence of S and L spaces lies in the properties of their finite powers. This gives some explanation as to why the existence of $\mathrm{S}$ and L spaces seem to be such similar hypotheses at first.

There are a number of results under $\mathrm{MA}_{\aleph_{1}}$ which limit the existence of $\mathrm{S}$ and $\mathrm{L}$ spaces.

\footnotetext{
${ }^{9}$ Here a space is a strong S (L) space iff all of its finite powers are S (L) spaces.
} 
Theorem $\left.7.2(9]\left(\mathrm{MA}_{\aleph_{1}}\right)\right)$. There are no strong $S$ or $L$ spaces.

Theorem $7.3\left([16]\left(\mathrm{MA}_{\aleph_{1}}\right)\right)$. There are no first countable L spaces.

Theorem $\left.7.4(2]\left(\mathrm{MA}_{\aleph_{1}}\right)\right)$. If $K$ is a compact space which contains an L space, then $K$ maps continuously onto $[0,1]^{\aleph_{1}}$.

Finally, Todorcevic proved that S spaces do not exist assuming PFA.

Theorem 7.5 (18 (PFA)). If a regular Hausdorff space is hereditarily separable, then it is hereditarily Lindelöf.

He also demonstrated that the existence of S and L spaces are different considerations.

Theorem 7.6 (21]). It is consistent with $\mathrm{MA}_{\aleph_{1}}$ that there are no $S$ spaces and yet that there is an $L$ space.

Until now, however, it remained unclear whether Todorcevic's methods could be used to prove that PFA implies there are no L spaces. I will now show that this is not the case - that there is an $\mathrm{L}$ space which can be constructed without appealing to additional axioms of set theory.

Definition 7.7. For each $\beta<\omega_{1}$, define $w_{\beta}: \omega_{1} \rightarrow \mathbb{T}$ by $w_{\beta}(\xi)=o(\xi, \eta)$ if $\xi<\beta$ and $w_{\beta}(\xi)=1$ otherwise. Let $\mathscr{L}$ be the collection $\left\{w_{\beta}: \beta<\omega_{1}\right\}$ viewed as a subspace of $\mathbb{T}^{\omega_{1}}$.

The " +1 " in the exponent of $o(\xi, \beta)$ is present so that $w_{\beta}(\xi)=1$ iff $\xi \geq \beta$. This will simplify the discussion at certain points. Notice that $\mathscr{L}$ is non-separable.

If $X \subseteq \omega_{1}$, then let $\mathscr{L}_{X}$ be $\left\{w_{\beta}\lceil X: \beta \in X\}\right.$ viewed as a subspace of $\mathbb{T}^{X}$. Since the map $w_{\beta} \mapsto w_{\beta}\left\lceil X\right.$ defined on $\left\{w_{\beta}: \beta \in X\right\}$ is a bijection, we will write $w_{\beta}$ for $w_{\beta}\left\lceil X\right.$ when referring to elements of $\mathscr{L}_{X}$. It should be emphasized, however, that while the above bijection is continuous, its inverse may not be. Hence, even with this identification, $\mathscr{L}_{X}$ should not be viewed as a subspace of $\mathscr{L}$.

We will now see that $\mathscr{L}_{X}$ is Fréchet. Let $\mathscr{U}$ be the collection of open $\operatorname{arcs}$ in $\mathbb{T}$ whose endpoints are roots of 1 and which do not contain 1 as an element. Let $\mathscr{F}$ be the collection of all sets of the form $\{w \in \mathscr{L}: w(\alpha) \in U\}$ where $U$ is an element of $\mathscr{U}$ and $\alpha$ is in $X$. Notice that $\mathscr{F}$ is point countable and that the topology on $\mathscr{L}_{X}$ is the smallest in which every element of $\mathscr{F}$ is clopen. The following theorem shows that $\mathscr{L}_{X}$ is always Fréchet.

Theorem 7.8. If $X$ is a set and $\mathscr{F}$ is a point countable, point separating family of subsets of $X$, then the topology on $X$ defined by declaring elements of $\mathscr{F}$ to be clopen is countably tight and has every countable subspace metrizable. In particular the topology is Fréchet.

Remark 7.9. I attribute this result to Zoltan Balogh. He once told me that "if there is an L space, then there is a countably tight one." While I never saw his proof, I think it is reasonable to assume this he may have proceeded along these lines.

Proof. That countable subspaces are metrizable follows from the assumptions and the well-known fact that regular Hausdorff second countable spaces are metrizable. To see that the space is countably tight, let $A \subseteq X$ have an accumulation point $x$ in $X$. Let $M$ be a countable elementary submodel of $H(\theta)$ for $\theta$ regular and 
large enough so that $X, \mathscr{F}, x$, and $A$ are all in $M$. It suffices to show that $x$ is an accumulation point of $A \cap M$.

Suppose this is not the case and let $U_{i}(i<k)$ and $V_{i}(i<l)$ be elements of $\mathscr{F}$ such that

$$
W=\bigcap_{i<k} U_{i} \backslash \bigcup_{j<l} V_{j}
$$

contains $x$ and is disjoint from $A \cap M$. Without loss of generality, we may assume that the empty set is not in $\mathscr{F}$. The important observation is this: if $V$ is in $\mathscr{F}$, then $V \cap M$ is non-empty iff $V$ is in $M$. This is because $\{V \in \mathscr{F}: y \in V\}$ is countable for all $y$ in $X$ and therefore a subset of $M$ whenever $y$ is in $M$ (by Facts 5 and 7). Hence each $U_{i}$ must be in $M$ since $x$ is in $U_{i}$ and $x$ is in $M$. Moreover, since we are only interested in having $W$ be disjoint from $A \cap M$, we may assume without loss of generality that each $V_{j}$ is in $M$. But then $W$ must be in $M$ and, by the elementarity of $M$, there must be an element of $W \cap A$ which is in $M$, a contradiction.

As mentioned above, if $X$ is uncountable, then $\mathscr{L}_{X}$ can be shown to be an $\mathrm{L}$ space using Theorem 5.6 and the methods of $[5$. I will prove the following theorem of independent interest and then derive this as a consequence.

Theorem 7.10. If $X, Y \subseteq \omega_{1}$ have countable intersection, then there is no continuous injection from any uncountable subspace of $\mathscr{L}_{X}$ into $\mathscr{L}_{Y}$.

Proof. Suppose for contradiction that such an injection $g$ does exist. Then $g$ is of the form $w_{\beta} \mapsto w_{f(\beta)}$ where $f: X_{0} \rightarrow Y$ is an injection for some uncountable $X_{0} \subseteq X$ which is, without loss of generality, disjoint from $Y$. For each $\xi<\omega_{1}$, let $\beta_{\xi}$ and $\zeta_{\xi}$ be elements of $X_{0}$ and $Y$ respectively such that $f\left(\beta_{\xi}\right)>\zeta_{\xi}$ and if $\xi<\xi^{\prime}$, then $\beta_{\xi}<\zeta_{\xi^{\prime}}$. Let $\Xi \subseteq \omega_{1}$ be uncountable such that for some open neighborhood $V$ in $\mathbb{T}, g\left(w_{\beta_{\xi}}\right)\left(\zeta_{\xi}\right)$ is not in the closure of $V$ whenever $\xi$ is in $\Xi$.

Applying the continuity of $g$ at $w_{\beta_{\xi}}$ to

$$
W_{\xi}=\left\{w \in \mathscr{L}_{Y}: w\left(\zeta_{\xi}\right) \notin \bar{V}\right\},
$$

there is a basic open neighborhood $U_{\xi}$ of $w_{\beta_{\xi}}$ such that $U_{\xi} \subseteq g^{-1} W_{\xi}$. Applying the $\Delta$-system lemma and the second countability of $\mathbb{T}$, there is an uncountable $\Xi^{\prime} \subseteq \Xi$, open neighborhoods $U_{i}(i<k)$ in $\mathbb{T}$, and $a_{\xi}$ in $[X]^{k}$ such that for all $\xi$ in $\Xi^{\prime}$ :

(22) $\left\{a_{\xi}: \xi \in \Xi^{\prime}\right\}$ is a $\Delta$-system with $\operatorname{root} a$;

(23) the set

$$
\left\{w \in \mathscr{L}_{X}: \forall i<k\left(w\left(a_{\xi}(i)\right) \in U_{i}\right)\right\}
$$

has $w_{\beta_{\xi}}$ as an element and is a subset of $U_{\xi}$;

(24) the inequality $\beta_{\xi}<f\left(\beta_{\xi}\right)$ does not depend on $\xi$;

(25) $\left|\zeta_{\xi} \cap a_{\xi}\right|$ does not depend on $\xi$.

Let $\mathscr{A}$ be the collection of all $a_{\xi} \cup\left\{\zeta_{\xi}\right\} \backslash a$ and $\mathscr{B}$ be the collection of all $\left\{\beta_{\xi}, f\left(\beta_{\xi}\right)\right\}$. Applying Theorem 5.6, it is possible to find $\xi<\xi^{\prime}$ in $\Xi^{\prime}$ such that for all $i<k$,

$$
\begin{gathered}
a_{\xi} \cup\left\{\zeta_{\xi}\right\}<\min \left(\beta_{\xi^{\prime}}, f\left(\beta_{\xi^{\prime}}\right)\right), \\
w_{\beta_{\xi^{\prime}}}\left(a_{\xi}(i)\right)=o\left(a_{\xi}(i), \beta_{\xi^{\prime}}\right) \in U_{i}, \\
g\left(w_{\beta_{\xi^{\prime}}}\right)=w_{f\left(\beta_{\xi^{\prime}}\right)}\left(\zeta_{\xi}\right)=o\left(\zeta_{\xi}, f\left(\beta_{\xi^{\prime}}\right)\right) \in V .
\end{gathered}
$$

But now $w_{\beta_{\xi^{\prime}}}$ is an element of $U_{\xi}$ even though $g\left(w_{\beta_{\xi^{\prime}}}\right)$ is not in $W_{\xi}$, contradicting our choice of $U_{\xi}$. This finishes the proof. 
Corollary 7.11. For every $X, \mathscr{L}_{X}$ is hereditarily Lindelöf.

Proof. If not, then $\mathscr{L}_{X}$ would contain an uncountable discrete subspace. It is then possible to find disjoint $Y, Z \subseteq X$ such that $\mathscr{L}_{Y}$ and $\mathscr{L}_{Z}$ each contain uncountable discrete subspaces. But any function from a discrete space to another discrete space is continuous, and we can then easily contradict Theorem 7.10 ,

Kunen has shown, however, that under $\mathrm{MA}_{\aleph_{1}}$ every L space contains an uncountable discrete subspace in one of its finite powers [9]. As might be expected, this happens at the first possible instance in our example.

Theorem 7.12. For every uncountable $X \subseteq \omega_{1}, \mathscr{L}_{X}{ }^{2}$ contains an uncountable discrete subspace.

Proof. This essentially follows from the next proposition which is of independent interest.

Proposition 7.13. The tree

$$
T(o)=\left\{o(\cdot, \beta)\left\lceil\alpha: \alpha \leq \beta<\omega_{1}\right\}\right.
$$

is Aronszajn.

Proof. Theorem 5.6 implies that $T(o)$ does not contain an uncountable branch. It suffices to prove that all levels of $T(o)$ are countable. For this, it is sufficient to show that $T\left(\varrho_{1}\right)$ and $T(L)$ have countable levels. In the case of $T\left(\varrho_{1}\right)$, this is the content of Remark 2.8. In order to see that $T(L)$ has countable levels, let $\alpha<\omega_{1}$ be given. Let $\beta<\omega_{1}$ be greater than $\alpha$. Using the compactness of $\alpha+1$ and Fact 2. select a finite set $F_{\beta} \subseteq \alpha+1$ containing 0 and $\alpha$ so that if $\gamma_{0}<\gamma$ are consecutive elements of $F_{\beta}$, then $L(\gamma, \beta)<L(\xi, \gamma)$ whenever $\gamma_{0}<\xi<\gamma$. It suffices to show that if

$$
L(\cdot, \beta)\left\lceil F_{\beta}=L\left(\cdot, \beta^{\prime}\right) \uparrow F_{\beta^{\prime}},\right.
$$

then

$$
L(\cdot, \beta)\left\lceil\alpha=L\left(\cdot, \beta^{\prime}\right)\lceil\alpha .\right.
$$

To see this, let $\xi$ be an arbitrary element of $\alpha \backslash F_{\beta}$ and pick $\gamma_{0}<\gamma$ in $F_{\beta}$ so that $\gamma_{0}<\xi<\gamma$. Applying Fact 1 we have

$$
L(\xi, \beta)=L(\xi, \gamma) \cup L(\gamma, \beta)=L(\xi, \gamma) \cup L\left(\gamma, \beta^{\prime}\right)=L\left(\xi, \beta^{\prime}\right) .
$$

To finish the proof of Theorem 7.12 , select a sequence $\left(\beta_{\xi}^{0}, \beta_{\xi}^{1}\right)$ indexed by an uncountable set $\Xi \subseteq \omega_{1}$ such that for each $\xi$ in $\Xi$ the following conditions are satisfied:

(26) $\beta_{\xi}^{0}<\beta_{\xi}^{1}$ are both elements of $X$;

(27) there is a fixed $\varepsilon>0$ such that

$$
\left|w_{\beta_{\xi}^{0}}\left(\beta_{\xi}^{0}\right)-w_{\beta_{\xi}^{1}}\left(\beta_{\xi}^{0}\right)\right|=\left|1-w_{\beta_{\xi}^{1}}\left(\beta_{\xi}^{0}\right)\right| \geq \varepsilon ;
$$

(28) if $\eta<\xi$ is in $\Xi$, then $\beta_{\eta}^{0}<\xi$;

(29) $w_{\beta_{\xi}^{0}}\left\lceil\xi=w_{\beta_{\xi}^{1}}\lceil\xi\right.$.

This is possible since $T(o)$ has countable levels. Now consider the open neighborhoods

$$
U_{\xi}=\left\{(u, v) \in \mathscr{L}_{X}:\left|w_{\beta_{\xi}^{0}}\left(\beta_{\xi}^{0}\right)-u\left(\beta_{\xi}^{0}\right)\right|+\left|v\left(\beta_{\xi}^{0}\right)-w_{\beta_{\xi}^{1}}\left(\beta_{\xi}^{0}\right)\right|<\varepsilon\right\}
$$


of $\left(w_{\beta_{\xi}^{0}}, w_{\beta_{\xi}^{1}}\right)$ in $\mathscr{L}_{X}^{2}$ for $\xi$ in $\Xi$. If $\eta<\xi$ are in $\Xi$, then by (27) $\left(w_{\beta_{\eta}^{0}}, w_{\beta_{\eta}^{1}}\right)$ is not in $U_{\xi}$ since $w_{\beta_{\eta}^{1}}\left(\beta_{\xi}^{0}\right)=1$. If $\xi<\eta$ are in $\Xi$, then by (28) $\beta_{\xi}^{0}<\eta$ and by (29)

$$
w_{\beta_{\eta}^{0}}\left(\beta_{\xi}^{0}\right)=w_{\beta_{\eta}^{1}}\left(\beta_{\xi}^{0}\right) \text {. }
$$

Consequently $\left(w_{\beta_{\eta}^{0}}, w_{\beta_{\eta}^{1}}\right)$ being in $U_{\xi}$ would contradict (27) via the triangle inequality. Hence $\left(w_{\beta_{\eta}^{0}}, w_{\beta_{\eta}^{1}}\right)$ is in $U_{\xi}$ iff $\eta=\xi$ and therefore $\left\{\left(w_{\beta_{\xi}^{0}}, w_{\beta_{\xi}^{1}}\right): \xi \in \Xi\right\}$ is an uncountable discrete subspace of $\mathscr{L}_{X}{ }^{2}$ as desired.

At this point, I will make the following conjecture.

Conjecture 4 (PFA). If $X$ is a non-separable regular Hausdorff space, then $X^{2}$ contains an uncountable discrete subspace.

While $\mathscr{L}$ is 0 -dimensional, its closure in $\mathbb{T}^{\omega_{1}}$ is very far from being 0 -dimensional.

Theorem 7.14. If $E \subseteq \mathscr{L}$ is uncountable, then the closure of $E$ in $\mathbb{T}^{\omega_{1}}$ contains a set of the form $\left\{f \in \mathbb{T}^{\omega_{1}}: f\lceil\alpha=\tau\}\right.$ for some $\alpha<\omega_{1}$ and $\tau$ in $\mathbb{T}^{\alpha}$. In particular, $\operatorname{cl}(E)$ is not 0-dimensional.

Remark 7.15. This example may be informative in studying the following problem of M. E. Rudin: Is it consistent that every locally connected perfect compact space is metrizable? See [4].

Proof. Let $E \subseteq \mathscr{L}$ be uncountable and let $T(E)$ be the set of all $\tau$ in $T(o)$ such that for uncountably many $\beta$ in $E, \tau$ is a restriction of $w_{\beta}$. If $\tau$ is in $T(E)$, let $E_{\tau}$ denote the set of all $w_{\beta}$ in $E$ such that $\tau$ is a restriction of $w_{\beta}$.

Claim 7.16. For each $\tau$ in $T(E)$ there is an $\alpha_{\tau}<\omega_{1}$ such that the projection map $\pi: \mathbb{T}^{\omega_{1}} \rightarrow \mathbb{T}^{\omega_{1} \backslash \alpha_{\tau}}$ sends the closure of $E_{\tau}$ onto $\mathbb{T}^{\omega_{1} \backslash \alpha_{\tau}}$.

Proof. Suppose for contradiction that this is not the case. For each $\xi<\omega_{1}$, select a basic open neighborhood $W_{\xi} \subseteq \mathbb{T}^{\omega_{1}}$ such that $W_{\xi} \cap E_{\tau}$ is empty and membership in $W_{\xi}$ depends only on coordinates greater than $\xi$. By refining the sequence $W_{\xi}(\xi<$ $\left.\omega_{1}\right)$ it is possible to find open neighborhoods $U_{i}(i<k)$ in $\mathbb{T}$ and an uncountable pairwise disjoint $\mathscr{A} \subseteq\left[\omega_{1}\right]^{k}$ such that if $a$ is in $\mathscr{A}$, then there is no $w_{\beta}$ in $E_{\tau}$ with

$$
w_{\beta}(a(i)) \in U_{i}
$$

for all $i<k$. Pairing $\mathscr{A}$ with $\mathscr{B}=\left\{\{\beta\}: w_{\beta} \in E_{\tau}\right\}$, we have a contradiction to Theorem 5.6.

Let $\delta<\omega_{1}$ be such that if $\tau$ is an element of $T(E)$ of height less than $\delta$, then $\alpha_{\tau}<\delta$. Since $T(o)$ has countable levels, such a $\delta$ exists. Since $T(E)$ is uncountable and downwards closed, there is a $\tau$ in $T(E)$ of height $\delta$. Put

$$
F=\bigcap_{\xi<\delta} \operatorname{cl}\left(E_{\tau \mid \xi}\right) .
$$

Notice that the projection of each $\operatorname{cl}\left(E_{\tau \uparrow \xi}\right)$ onto the set of coordinates $\omega_{1} \backslash \delta$ is onto $\mathbb{T}^{\omega_{1} \backslash \delta}$. Since the fibers of this map are compact and since the intersection is decreasing, $F$ must project onto $\mathbb{T}^{\omega_{1} \backslash \delta}$. Since every element of $F$ is an extension of $\tau$,

$$
F=\left\{f \in \mathbb{T}^{\omega_{1}}: f \text { extends } \tau\right\}
$$

is the desired subset of $\operatorname{cl}(E)$. 
The proof of Theorem 7.3 can be used to prove the following.

Theorem 7.17. For every $X \subseteq \omega_{1}$, no uncountable subspace of $\mathscr{L}_{X}$ is first countable.

Proof. The proof of Theorem 7.3 actually shows that if an L space is first countable, then there is a c.c.c. forcing which destroys it. It is easily checked that every uncountable subspace of $\mathscr{L}_{X}$ is an $\mathrm{L}$ space in every c.c.c. forcing extension.

We also have the following consequence of Proposition 7.13 .

Theorem 7.18. If $f$ is a continuous function from a subspace of $\mathscr{L}$ into a metric space, then the range of $f$ is countable.

Proof. Since $\mathscr{L} \subseteq \mathbb{T}^{\omega_{1}}$ is hereditarily Lindelöf and $f$ is continuous, $f$ depends only on a countable set of coordinates. The conclusion of the theorem now follows from Proposition 7.13

Theorem 7.19. Any basis for the uncountable regular Hausdorff spaces must have cardinality greater than $\aleph_{1}$.

Proof. The first conclusion follows from Theorem 7.10 and the observation that there is an almost disjoint family of uncountable subsets of $\omega_{1}$ of cardinality $\aleph_{2}$.

I will close this paper by referring the reader to [4] where a number of open problems related to this line of research are collected and discussed.

\section{ACKNOWLEDGMENTS}

I would like to thank Boban Veličković for his careful reading of early drafts of this paper. The clarity of the present arguments owe much to Todorcevic's and Veličković's suggestions. A number of other people - too many to name - have also made the generous contribution of reading the paper and offering suggestions. Finally, special thanks are due to the diligent referees for their careful reading of the paper.

\section{REFERENCES}

[1] J. W. S. Cassels. An introduction to Diophantine approximation. Cambridge Tracts in Mathematics and Mathematical Physics, No. 45. Cambridge University Press, New York, 1957. MR0087708 (19:396h)

[2] D. H. Fremlin. Consequences of Martin's Axiom. Cambridge University Press, 1984. MR0780933 (86i:03001)

[3] G. Gruenhage. Perfectly normal compacta, cosmic spaces, and some partition problems. In Open problems in topology, pages 85-95. North-Holland, Amsterdam, 1990. MR1078642

[4] G. Gruenhage and J. Tatch Moore. Perfect compacta and basis problems in topology. In Open Problems in Topology II. In preparation, Sept. 2005.

[5] A. Hajnal and I. Juhász. On hereditarily $\alpha$-Lindelöf and hereditarily $\alpha$-separable spaces. Ann. Univ. Sci. Budapest. Eötvös Sect. Math., 11:115-124, 1968. MR0240779 (39:2124)

[6] T. Jech. Multiple forcing, volume 88 of Cambridge Tracts in Mathematics. Cambridge University Press, Cambridge, 1986. MR0895139(89h:03001)

[7] I. Juhász. A survey of $S$ - and $L$-spaces. In Topology, Vol. II (Proc. Fourth Colloq., Budapest, 1978), volume 23 of Colloq. Math. Soc. János Bolyai, pages 675-688. North-Holland, Amsterdam, 1980. MR0588816 (81j:54001)

[8] L. Kronecker. Näherungsweise ganzzahlige Auflösung linearer Gleichungen. S.-B. Preuss. Akad. Wiss., 1884. S.-B. Preuss. Akad. Wiss. 1179-83, 1271-99, Werke III (1), 47-109. 
[9] K. Kunen. Strong $S$ and $L$ spaces under $M A$. In Set-theoretic topology (Papers, Inst. Medicine and Math., Ohio Univ., Athens, Ohio, 1975-1976), pages 265-268. Academic Press, New York, 1977. MR0440487 (55:13362)

[10] K. Kunen. An introduction to independence proofs, volume 102 of Studies in Logic and the Foundations of Mathematics. North-Holland, 1983. MR0756630 (85e:03003)

[11] Dj. Kurepa. Ensembles ordonnés et ramifiés. Publ. Math. Univ. Belgrade, 4:1-138, 1935.

[12] J. Roitman. Basic $S$ and L. In Handbook of set-theoretic topology, pages 295-326. NorthHolland, Amsterdam, 1984. MR0776626 (87a:54043)

[13] M. E. Rudin. $S$ and $L$ spaces. In Surveys in general topology, pages 431-444. Academic Press, New York, 1980. MR0564109 (81d:54003)

[14] W. Sierpiński. Sur l'equivalence de trois propriétés des ensembles abstraits. Fundamenta Mathematicae, 2:179-188, 1921.

[15] M. Suslin. Problème 3. Fund. Math., 1:223, 1920.

[16] Z. Szentmiklóssy. S spaces and L spaces under Martin's Axiom. In Topology, volume 23 of Coll. Math. Soc. Janos Bolyai, pages 1139-1145. North-Holland, 1980. Fourth Colloq., Budapest 1978. MR0588860 (81k:54032)

[17] P. L. Tchebychef. Sur une question arithmétique. Denkschr. Akad. Wiss. St. Petersburg, 1(4):637-84, 1866.

[18] S. Todorcevic. Forcing positive partition relations. Trans. Amer. Math. Soc., 280(2):703-720, 1983. MR0716846 (85d:03102)

[19] S. Todorcevic. Partitioning pairs of countable ordinals. Acta Math., 159(3-4):261-294, 1987. MR.0908147 (88i:04002)

[20] S. Todorcevic. Oscillations of real numbers. In Logic colloquium '86 (Hull, 1986), volume 124 of Stud. Logic Found. Math., pages 325-331. North-Holland, Amsterdam, 1988. MR0922115 (89c:04001)

[21] S. Todorcevic. Partition Problems in Topology. Amer. Math. Soc., 1989. MR0980949 (90d:04001)

[22] S. Todorcevic. A classification of transitive relations on $\omega_{1}$. Proc. London Math. Soc. (3), 73(3):501-533, 1996. MR.1407459 (97k:04001)

[23] S. Todorcevic. Basis problems in combinatorial set theory. In Proceedings of the International Congress of Mathematicians, number Extra Vol. II, pages 43-52, 1998. MR1648055 (2000c:03039)

[24] S. Todorcevic. Coherent sequences. In Handbook of Set Theory. North-Holland (forthcoming).

[25] J. W. Tukey. Convergence and uniformity in topology. Princeton Univ. Press, 1940. MR0002515 (2:67a)

[26] P. Vojtáš. Generalized Galois-Tukey-connections between explicit relations on classical objects of real analysis. In Set theory of the reals (Ramat Gan, 1991), pages 619-643. Bar-Ilan Univ., Ramat Gan, 1993. MR.1234291 (95e:03139)

[27] P. Zenor. Hereditary $\mathfrak{m}$-separability and the hereditary $\mathfrak{m}$-Lindelöf property in product spaces and function spaces. Fund. Math., 106(3):175-180, 1980. MR0584491 (82a:54039)

Department of Mathematics, Boise State University, Boise, Idaho 83725

E-mail address: justin@math.boisestate.edu 